\title{
Interference in axisymmetric synthetic jet actuator
}

\author{
Emil Smyk ${ }^{*}$ \\ UTP University of Science and Technology, Faculty of Mechanical Engineering, Control Group, Poland
}

\begin{abstract}
The paper presents research of phenomena of mechanical wave interference during generate synthetic jets in actuator with two executive elements (speakers). Two speakers work with the same or other phase using the phenomenon of mechanical wave interference (sound wave). In effect the volume of ingestion and expulsion flow is a sum of two speakers' work. In this paper is presented analysis of operation so constructed axisymetric actuator and influence of phase shift - between work of the speakers - on actuator's operation.
\end{abstract}

\section{Introductions}

Synthetic jets are formed by periodically suction and blowing of fluid to some chamber by nozzle. Device to produce synthetic jets is named synthetic jet actuator. It is realized by replacement one of actuator's wall by movable element. Depending on the method of accomplishing the movement of wall, can distinguish piezoelectric, electromagnetic, acoustic and mechanical synthetic jet actuators. Example of synthetic jet actuator and idea of synthetic jets forming are shown in fig. 1.

Name of "synthetic jet" was firstly used in [1]. It is to suggest that the flow is synthesised by creation of the succeeding vortex rings. It is worth of noted that because time- averaged flow by a nozzle of synthetic jet actuator is zero, the same volume of flow is ingestion and expulsion, synthetic jet are named zero-net massflux (ZNMF) jet. Synthetic jet actuator doesn't need additional fluid supply to correct functioning, what distinguishes synthetic jet from continuous jets.

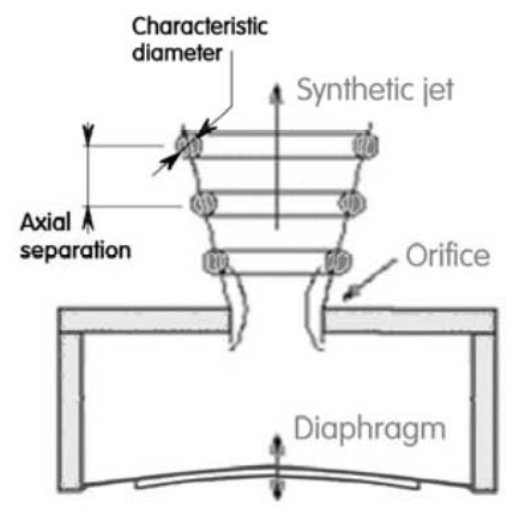

Fig. 1. Example of the idea of synthetic jets forming - from [2]

Property of synthetic jet are the reason of widespread interesting theme of their application. They are used in cooling applications, chemical engineering, active flow control etc. Particularly active flow control is a synthetic jet's applications field which is dynamically developed, demonstrated by a large number of papers appearing in recent years [2-6].

Interest of SJ widened not only knowledge that issue [7-10], but conducive to creating new synthetic jet's applications [11]. Interesting method of generation synthetic jet was presented in [12, 13, 14]. In all of paper actuators was construct by replacement two of opposed walls by a diaphragm of speakers (fig. 2). Such construction of actuators should increase velocity of synthetic jet.

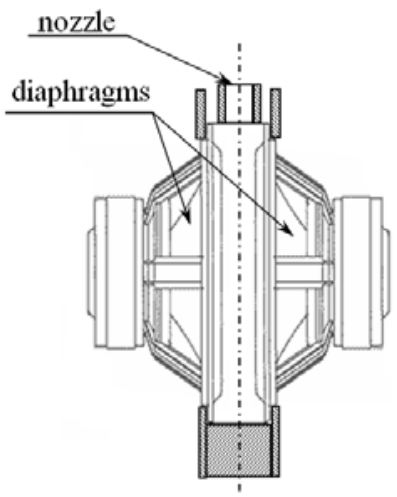

Fig. 2. Example of synthetic jet actuator with two speakers

Two speakers operate with the same phase using the phenomenon of mechanical wave interference (sound wave). In effect the volume of ingestion and expulsion flow is a sum of two speakers' operation. It must be noted that in [12] speakers works in this same way, what is provided by actuators construction. However, in [13] can be seen that work of one of speakers is suppressed. This is caused by putting the speaker in closed chamber. During his work preassure behind speaker periodically decreases (when fluid is expulsion from actuator by movement speaker diaphgram 'forward') and increses (when fluid is ingestion to actuator by movement speaker diaphgram 'backward').

Although in $[12,13,14]$ was used this specifical type of actuators, they do not include information about

* Corresponding author: emil.smyk@,utp.edu.pl 
interferenc of wave, and how it influence at parameters of synthetic jet.

The aim of this work is investigate how phenomene of interferenc influeces on synthetic jets.

\section{Parameters}

The synthetic jets are produced by periodically expulsion and ingestion of fluid. Therefore, their velocity can't be determined in the same way as continuous jets velocity. The time-mean velocity $U_{0}$ define from the orifice centerline at the axis:

$$
U_{0}=\frac{1}{T} \int_{0}^{T_{E}} u_{0} d t
$$

Where $T$ is time period ( $T=1 / f$, where $f$ is frequency), $T_{E}$ is time of flow expulsion $\left(T_{E}=T / 2\right)$, and $u_{0}$ is velocity of flow.

Another parameters used in case of synthetic jet actuator is nondimensional stroke ratio $L / d$ and Reynolds number $R e$. They have been identified as key actuator operational parameters that influence synthetic jet [1]. Stroke ratio $L / d$ is a proportion between length and diameter of expulsion fluid volume (fig. 3). So it is ratio of distance over which the fluid is blown and the size of nozzle. Stroke ratio $L / d$ can be calculated from:

$$
\alpha \frac{\pi D^{2}}{4} \Delta=\frac{\pi d^{2}}{4}
$$

Where $\alpha$ is the fraction of the volume displaced by an imaginary piston undergoing a peak to peak deflection of $\Delta . D$ is inner diameter of actuator and $d$ is diameter of nozzle.

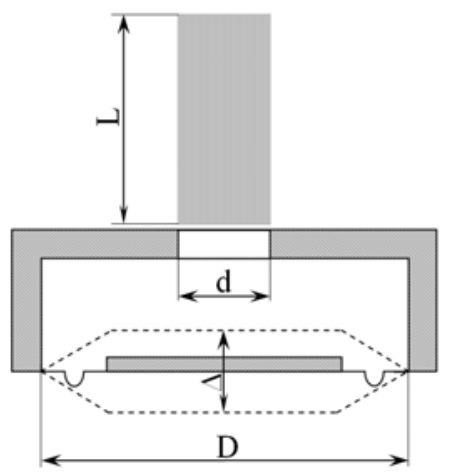

Fig. 3. Schema of actuator, where volume of fluid displaced by the diaphragm is ejected through the orifice in the form of slug

The definitions of the nondimensional parameters Reynolds numbers $R e$ can be introduced as follows:

$$
R e=\frac{U_{0} d}{v}
$$

Where $v$ is kinematic viscosity of fluid.

It must be noted that these formulas can be used for incompressible fluids. In case of compressible fluids (example air) this formulas can be used for Mach number less than 0.3 . In such a case the error should be less than 3\%. Mach number can be calculated from:

$$
M a=\frac{u}{c}
$$

Where $u$ is local flow velocity fluid (gas), and $c$ is speed of sound in the medium.

\section{Research subject}

Aim of the study is examination of the application of interference phenomena to synthetic jet. Based on the work $[12,13]$ was made synthetic jet actuator presented in fig. 4.

Actuator consist of two opposite speakers Monacor SP-6/8SQ mounted on a metal ring. On the circuit therefore (ring) was done 24 holes in diameter $\phi 3$. On ring are attached two sleeves so as to create between them a gap of $0.5 \mathrm{~mm}$. One of sleeve creates from the back of the speaker closed chamber. Its task is suppression of speaker operation. By this actuator structure, speakers generates other wave, through the same power supply.

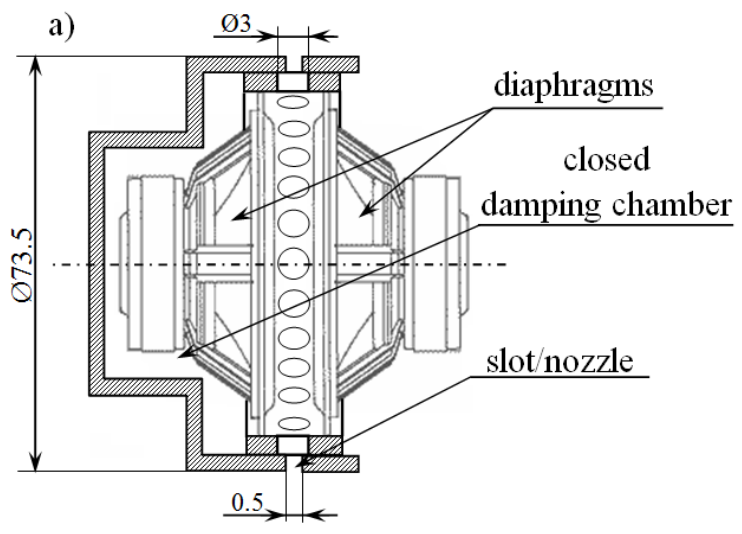

b)

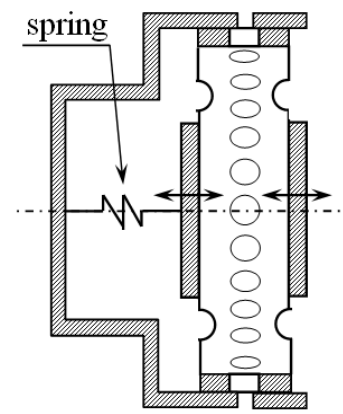

Fig. 4. Schema of synthetic jet actuator a) drawing, b) physical model

In fig. $4 \mathrm{~b}$ was showed physical model of synthetic jet actuator. In this model closed chamber on the left is replaced by spring. The restoring force of spring results from the chamber volume changes (the mass of air in chamber is constant). Bellow was presented analysis of spring properties. The analysis is based on fig. 5. 
To present the closed chamber as spring is needed the comparison of forces acting on the actuator diaphragm in the initial and final state. On the fig. 5 is presented model used to this analysis. The constant volume of air closed in chamber behind the speaker presses the diaphragm with forces $F_{0}$. It is due to air pressure. If the chamber is presented as a cylinder with fixed base area $A$ (area of speaker diaphragm) and a variable height $l$ ( $l_{0}$ is a initial height) then force $F_{0}$ can be calculated from ideal gas law:

$$
p_{0} l_{0} A=R T
$$

where $R$ is a universal gas constant $(R=8,314 \mathrm{~J} /(\mathrm{mol} \cdot \mathrm{K})), T$ is a temperature in Kelvin. Then converted equation (5):

$$
\begin{gathered}
p_{0} A=\frac{R T}{l_{0}} \\
F_{0}=\frac{R T}{l_{0}}
\end{gathered}
$$

However, if the membrane is ejected from a distance $\Delta l$ force will decrease and will equal:

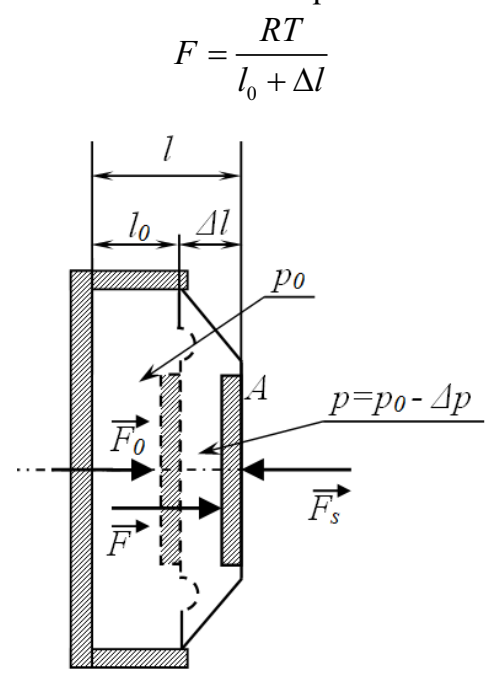

Fig. 5. Simplified model of the speaker with a closed chamber

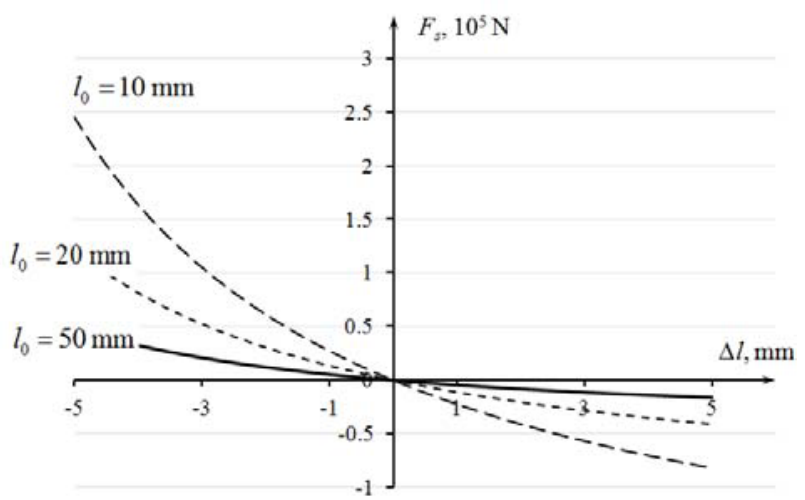

Fig. 6. Force characteristic for different chamber length $l_{0}$

The force acting on the diaphragm decreases when the chamber volume increases (or increases if the volume of chamber decreases). The change of this force can by presented by force $F_{s}$ which resist movement of the diaphragm.

$$
\begin{gathered}
F_{s}=F-F_{0} \\
F=\frac{R T}{l_{0}+\Delta l}-\frac{R T}{l_{0}} \\
F=-R T \frac{\Delta l}{l_{0}\left(l_{0}+\Delta l\right)}
\end{gathered}
$$

Analyzing the equation (10) can be seen that the closed chamber behind the speaker can be treated as a non-linear spring, which modulus of elasticity is dependent on the volume of the chamber (the bigger $l$ the lower the modulus of elasticit). Sample plots the resilient force of the spring shown in fig. 6 .

The phenomena of mechanical wave interference occurs in the effect of the imposition a two wave with sundry amplitude or phase. Therefore test actuator was powered by arbitral waveform generator Rigol DG4162, with possibility of changing phase of current supply, for each from two supply channel. Electrical power was identified by measuring the voltage and current supply, and set by stereo amplifier SeoUm $\mathrm{Pa}-940 / 2$. Electric scheme of actuator connection was showed in fig. 7 .

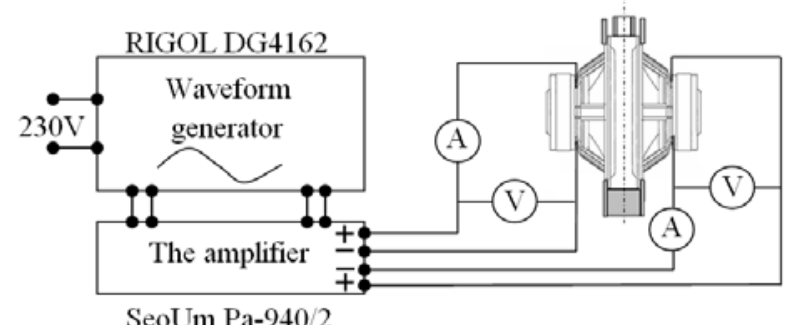

Fig. 7. Electric scheme of the actuator connection

All measurements of synthetic jet velocity was done by using hot-wire anemometer. Anemometer contained probe 55P16 connected through two modules MiniCTA and NI9215 with personal computer. Hot-wire probe was placed two millimeter from nozzle actuator in the nozzle axis. Measurements was carried out by temperature $T=22^{\circ}$ and pressure $p=1010 \mathrm{hPa}$.

\section{Results}

Before the main tests, there were carried out preliminary tests of the actuator. During primary testing, it was determined characteristic frequency of actuator. Then it was examined the operation of the synthetic jet actuator when power is one of the speakers. Furthermore examined how phase shift $\Delta \varphi$ of speakers work influence on synthetic jet velocity. In main tests, it was determined operating characteristic of actuator by different phase shift $\Delta \varphi$ of speakers operation.

It must be noted that as phase shift $\Delta \varphi=0^{\circ}$ understands the situation when both speakers operate in the same phase (example both speakers perform a movement forward and they push fluid from the actuator). 


\subsection{Primary tests}

The first step of tests was designation of characteristic frequency of actuator. It was identified as $f=147 \mathrm{~Hz}$. In the time of all further studies, actuator was powered by electric current with such frequency.

Then it was test whether phase shift of speakers operation has a influence on synthetic jet actuator. For this purpose was measured synthetic jet velocity for different phase shift $\Delta \varphi$ by a power supply $P=3 \mathrm{~W}$. The experiment data and sinusoidal approximation was showed in fig. 8 .

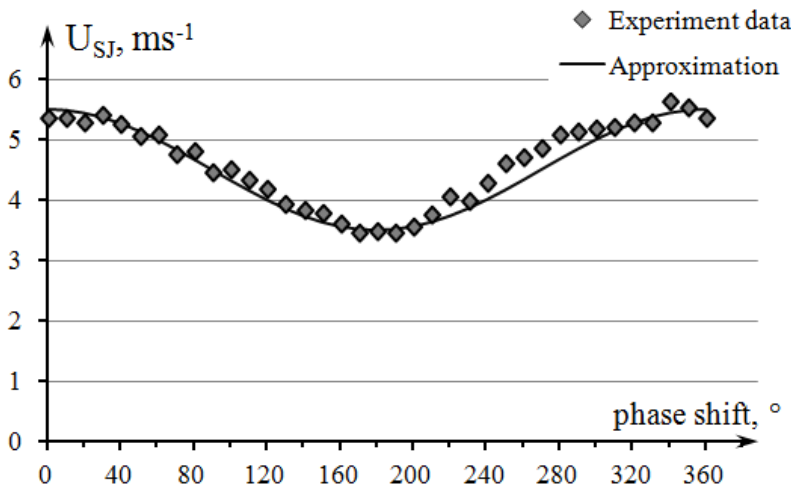

Fig. 8. Influence of phase shift on synthetic jet velocity for power supply $P=3 \mathrm{~W}$

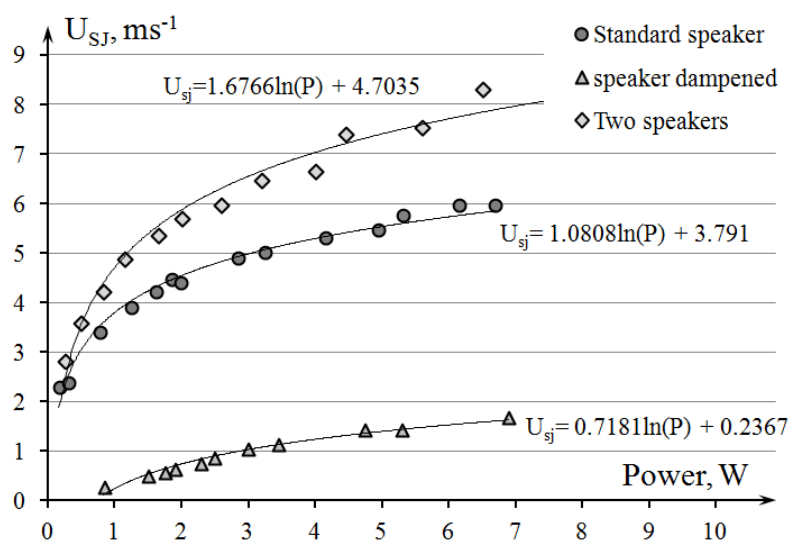

Fig. 9. Operating characteristic of actuator for speakers working separately and together

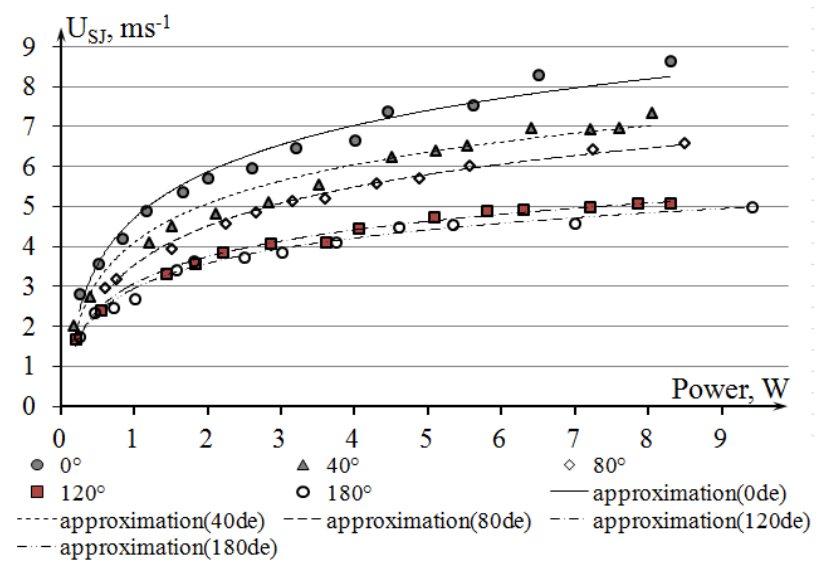

Fig. 10. Actuator characteristic for selected phase shift of speakers operation.

The next step was defined the operating characteristic for actuator by supply speakers separately and together.
The purpose of these measurements is also to determine whether the closed damping chamber has effect on the loudspeaker operation in the actuator. Characteristic of actuator is presented in fig. 9.

\subsection{Main tests}

The main part of the test is designate characteristic of actuator for different phase shift $\Delta \varphi$ of speakers operation. Fig. 10 presents actuator characteristic for selected phase shift $\Delta \varphi$ and logarithmic approximation of experimental data. It must be noted that $U_{S J}$ is a selected resultant of velocity and can't be used (in this case) to determine whether interference in actuator can be treated in a standard way.

In order to check how interference occurs in actuator, on the basis of measured velocity has been designated course of velocity in the actuator slot (fig. 4). The velocity change in the slot is a sine wave at a given frequency $(f=147 \mathrm{~Hz})$ but unknown amplitude $U_{M A X}$. This amplitude was calculated as average of maximum velocity from the measurements.

Then compare the amplitude waveforms (depends of electrical power $P$ ) calculated for real data and theoretical interference waves generated by speakers. Comparison of theoretical and experimental data is presented in fig. 11.

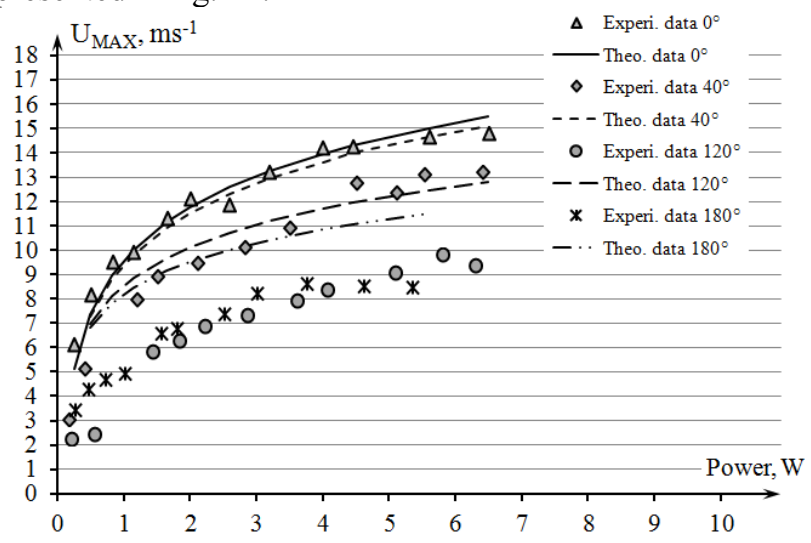

Fig. 11. Experimental and theoretical amplitude of air velocity in actuator slot

\section{Discussion of results}

Fig. 8 presents change of synthetic jet velocity depending of phase shift $\Delta \varphi$ of speakers. Trend of this change is as expected and can be approximated by sine wave. It exhibits some asymmetric however, it is negligibly small. This allowed for reduction of measurements (phase shift range between $\Delta \varphi=0^{\circ}$ to $\Delta \varphi=180^{\circ}$ ).

The next step of primal measurements was determination of operating characteristics of the actuator. It is presented in fig. 9. The damping chamber used in actuator has significant influence on operation of speaker. It is worth remembering that characteristic 'standard speaker' and 'dampened speaker'(fig. 9) is operating characteristic of the same speaker(the same model), but working in different conditions. The speaker with damping chamber generates synthetic jet only 
the power $P=1 \mathrm{~W}$, while the speaker without suppression produces synthetic jet from $P=0.2 \mathrm{~W}$.

As it was excepted the velocity, when powered two speakers, was greater then, when powered one speaker. It must be noted that, when low power, the synthetic jets are generated mainly by speaker without suppression. However, the influence of interference, occurring in actuator, is visible even for power less than $P=1 \mathrm{~W}$.

The hitherto measurements shows that the application of actuators with two executive elements is preferred because of increased of increased velocity of synthetic jet. In case of works [12,13] applications this actuator improved properties of test object without magnification of size.

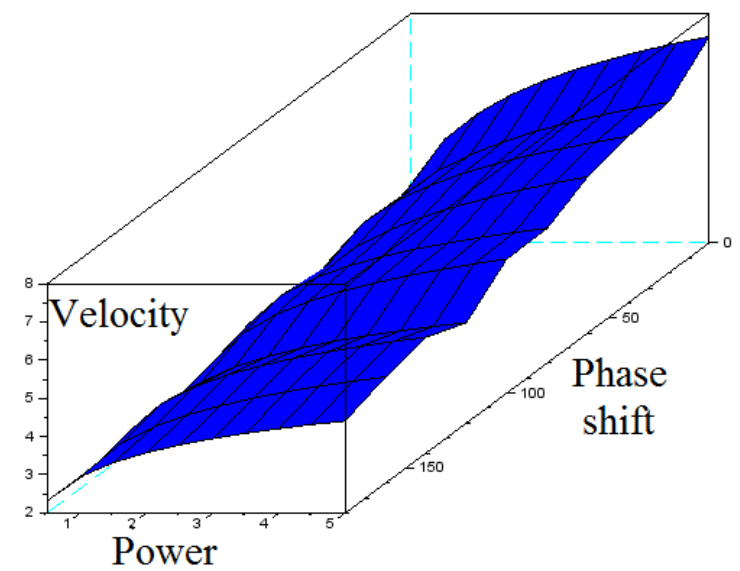

Fig. 12. Solution family of actuator operating characteristic

The next measurements involved in determining of operating characteristic of actuator with other phase shift $\Delta \varphi$. This solution helped designate the plane, which presents dependence between synthetic jet's velocity $U_{S J}$ and parameters of supply (power $P$ and phase shift $\Delta \varphi$ of speakers operation ). This dependence is showed in fig. 12. Examples operating characteristic are presented in fig. 10.

Fig. 12. presents some dependence between velocity and phase shift and power $\left(U_{S J}(P, \Delta \varphi)\right)$. As can be seen function $U_{S J}(P, \Delta \varphi)$ have a maximum with $\Delta \varphi=0^{\circ}$ (for specific power). This results is compatible with theory.

The above fact and data don't give sufficient information about the phenomena of interference in actuator. For this purpose was appointed the velocity amplitude $U_{M A X}$ in slot of the actuator (fig. 4). $U_{M A X}$ was designated as the average of maximum velocity of six next expulsion. It was calculated in case when speakers operated separately and together, whit other phase shift $\Delta \varphi$ and power $P$. Theoretical value of amplitude $U_{\text {MAX }}$ was calculated through addition two sine wave, first with velocity amplitude $U_{M A X}$ of damped speaker, and second of unsuppressed speaker, and with specified phase shift $\Delta \varphi$. The results are presented in fig. 11 .

It can be seen that theoretical and experimental velocity amplitudes $U_{M A X}$, with phase shift $\Delta \varphi=0^{\circ}$, are the same. But the greater phase shift $\Delta \varphi$ between speakers the greater different between experimental and theoretical data. Even for phase shift $\Delta \varphi=40^{\circ}$ difference between data is big. It is connected with uncorrected operation of actuator.

Speakers, which operate in different phase have tend to interfere with their mutual actions. Measurements shows increasing of turbulent of generated synthetic jet with simultaneous drop of velocity. It is undesirable effect. Moreover noted that the actuator operated with phase shift $\Delta \varphi$ is very sensitive on time of switch on.

Although, the speakers was powered by current with steady shape shift $\Delta \varphi$, the actuator generated synthetic jet on different velocity if the speakers wasn't switch on in the same time. It means that executive, elements in synthetic jet actuator which used phenomena of interference, have strong damping properties to each other if they don't operate in the same phase. It needs to be heighted that all measurements was carried out for speakers switched on in the same time. In case speaker switching at different times it happened that actuator produce synthetic jet on low-amplitude $\left(U_{M A X}=2 \mathrm{~m} \cdot \mathrm{s}^{-1}\right)$ even for high power $(P=6 \mathrm{~W})$. It mean that acoustic actuator using the interference phenomena is very sensitive on the desynchronization of speakers. Moreover, it should be noted that sensitivity is the greater the higher is the phase shift.

\section{Conclusion}

Presented results confirmed the reasonableness of the use of the actuator with two executive element. Moreover, the phenomena of interference, in produce of synthetic jet, was confirmed and discuss.

Additionally, in experiment was used closed damping chamber behind one of speakers. The chamber has significant and adverse influence on work of speaker and so on synthetic jet generated by him.

\section{Acknowledgements}

Scientific work financed from the budget for science in the years2015/2018 as a research project within the program under the name "Diamond Grant ".

\section{References}

1. B.L. Smith, A. Gezler, The formtion and evoluation of synthetic jets, Phisic of Fluids 31, p. 2281 (1998)

2. H. Tang, S. Zhong, Incompressible flow model on synthetic jet actuator, AIAA J. 44, p. 908 (2006)

3. D. Tamburello, A. Michael, Active control of a free jet using a synthetic jet, Int. J. of Heat and Fluid Flow, 29(4), p. 967-984 (2008)

4. D. Tamburello, M. Amitay, Three-dimensional interactions of a free jet with a perpendicular synthetic jet, J. of Turbul. 8(35), pp.1-22 (2007)

5. L.G. Pack, A. Seifert, Periodic excitation for jet vectoring and enhanced spreading, J. of Aircraft, 38(3), pp. 486-495 (2001) 
6. S.A. Davis, A. Glezer, The manipulation of largeand small-scales in coaxial jets using synthetic jet actuators, AIAA Paper 2000-0403 (2000)

7. P. Krejčí, Z. Trávníček, V. Tesař, J. Kordík, M. Pavelka, Frequency Characteristics of an Annular Synthetic Jet Actuator of Large Inner Diameter, Proceedings of Conf. 'Experimental Fluid Mechanics 2007' (2007)

8. Z. Travnicek, Z .Broucková, J. Kordík, Formation criterion for axisymmetric synthetic jets at high Stokes numbers, AIAA J. 50(9), pp. 2012 (2012)

9. V. Tesař, K. Peszynski, Experiments with synthetic jet for detecting potential terrorists, J. of Pol. CIMAC (2010)

10. P. Gil, P. Strzelczyk, Performance and efficiency of loudspeaker driven synthetic jet actuator, Exp. Therm. and Fluid Science 78, pp.163-174 (2016)
11. V. Tesař, K. Peszynski, Synthetic jet applied to detect potential terrorists, J. of Pol. CIMAC (2010)

12. V. Tesař, M. Pavelka, E. Smyk, K. Peszyński, Control of flow separation by vestigial" synthetic jet, Colloq. Fluid Dyn. (2014)

13. J. Kordik, T. Zdeněk M. Pavelka, Energetic efficiencies of synthetic and hybrid synthetic jet actuators driven by electrodynamic transducers, Exp. Therm. and Fluid Science 69, pp. 119-126 (2015)

14. V. Tesař, Z. Broučková, J. Kordik, Z. Trávníček, K. Peszyński, Valves with flow control by synthetic jets, EPJ Web of Conf. 25, EDP Sciences (2012) 